\title{
Indicadores de contaminación visual y sus efectos en la población
}

\section{(Visual pollution Indicators and its Effects on Population)}

\author{
Valeria Fuentes Correa ${ }^{1}$, Anita Argüello Mejía ${ }^{1}$
}

\begin{abstract}
Resumen:
Uno de los sectores donde se puede distinguir un aumento de la actividad comercial es la parroquia de Cotocollao, ubicada en el Distrito Metropolitano de Quito. Este desarrollo comercial ha significado un incremento de la publicidad exterior del sector, lo que supone una fuente de contaminación para el lugar. La presente investigación tiene como objetivos estimar los niveles de contaminación visual en un sector de la parroquia Cotocollao, y los efectos que esta tiene en la población, proponer indicadores de medición, analizar los puntos críticos que tiene el sector con respecto a la contaminación visual existente y el cumplimiento de la normativa local con respecto a la publicidad exterior fija en el sector. La metodología utilizada se basó en la propuesta de indicadores fabricados con base a la legislación local vigente, los cuales son planteados para realizar un análisis cuantitativo de este tipo de contaminación y así estimar los niveles existentes; y la aplicación de encuestas en la población del lugar para realizar un análisis cualitativo de los efectos de este tipo de contaminación. Los niveles estimados de contaminación visual presente en dicho sector han sido Medio y Medio-Alto en su mayoría y los efectos que este tipo de contaminación ha tenido en la población son cansancio visual, dolor de cabeza, estrés, obstrucciones visuales, entre otros.
\end{abstract}

Palabras clave: contaminación visual, Efectos en las personas, Indicadores, Publicidad.

\begin{abstract}
:
One of the areas where you can distinguish an increase in business activity is Cotocollao parish, located in the Metropolitan District of Quito, this commercial development has meant an increase in the outdoor advertising, which is a source of visual pollution. The aims of this investigation are estimate levels of visual pollution in an area of Cotocollao parish, and its effects on the population, propose indicators measuring, analyzing critical points that the area has with respect to the visual pollution and compliance with respect to the outdoor advertising set in the sector. Methodology is based on the proposal of indicators made based on the research conducted and local regulations, which are proposed for a quantitative analysis of this type of contamination and thus estimate existing levels; and implementation of surveys on the population of the place to make a qualitative analysis of the effects of this pollution. Estimated levels of visual pollution present in this place were the Middle and Upper-Middle mostly, and effects that this pollution has had on the population are eyestrain, headaches, stress, visual obstructions, among others.
\end{abstract}

Keywords: Visual Pollution, People Effects, Indicators, Publicity. 


\section{Introducción}

Existe varios tipos de contaminación en el ambiente, siendo los más conocidos y ampliamente analizados, los ocasionados a los tres grandes recursos: agua, aire y suelo; sin embargo, está presente también la contaminación urbana muy poco investigada, en la que se incluye la contaminación visual. Existen varios trabajos sobre temas relacionados a esta contaminación en varios países, siendo notable la falta de información al respecto, especialmente sobre estimaciones de los niveles de contaminación visual en el país, sus efectos en la población, así como métodos de medición. La investigación acerca de contaminación visual es importante, ya que puede existir un incremento de este tipo de contaminación y a futuro se podrán observar las consecuencias que traen la misma.

El objetivo principal de la presente investigación fue la estimación de los Niveles de contaminación visual en un sector de la parroquia Cotocollao ubicado en el Sector Zonal "La Delicia" y sus efectos en la población, mediante el establecimiento de indicadores de contaminación visual en un sector de la parroquia Cotocollao, basado en la normativa local e identificando los puntos críticos de contaminación visual en el sector. Esta investigación, contribuye con la estimación de los índices de contaminación visual en el sector de estudio y constituye de base para realizar otros estudios en varios sectores de la ciudad.

Existen varios autores que definen a la contaminación visual, siendo: contaminación visual es el cambio o desequilibrio en el paisaje, ya sea natural o artificial, que afecta las condiciones de vida y las funciones vitales de los seres vivos (Jérez Paredes, 2007). Jérez hace hincapié en la afección directa a la población; mientras que Méndez, se refiere a la afectación visual en el paisaje: Se define a la contaminación visual como el abuso de ciertos elementos "no arquitectónicos" que alteran la estética, la imagen del paisaje rural o urbano (Méndez Velandia, 2013). "El impacto en la imagen y fisonomía del entorno urbano causado por la acumulación de materia prima, productos, desechos, abandono de edificaciones y bienes materiales, así como violación en las densidades y características físicas de publicidad" (Méndez Velandia, 2013).

Como se puede observar, existen varias definiciones de contaminación visual, las mismas que, de manera general, se traducen a: contaminación visual es un tipo de contaminación de carácter urbano que es ocasionada por el uso excesivo de varios elementos ajenos al ambiente que alteran la estética y la imagen del paisaje ya sea natural o artificial, que afecta tanto a las condiciones, calidad de vida y las funciones vitales de los seres vivos.

Este tipo de contaminación se puede encontrar en los espacios públicos, por lo que es necesario tener una definición del mismo. Se entenderá como Espacio Público, el espacio de dominio público o privado en el que la publicidad exterior colocada es visible; incluyendo el espacio privado susceptible a la misma y, el espacio de servicio general en los que la publicidad colocada sea 
visible desde el espacio público (art. 3 Ordenanza 0330 del Municipio del Distrito Metropolitano de Quito, 2010).

Para la existencia de este tipo de contaminación existen varios focos: los espacios interiores, cables aéreos y antenas, estilos arquitectónicos mezclados, basureros, grafitis y pintadas, árboles y la publicidad exterior (Méndez Velandia, 2013). En el Sector Zonal “La Delicia”, el incremento de actividad comercial ha tenido un crecimiento gradual por lo que supone un aumento en publicidad trayendo consigo indicios de contaminación visual, por consiguiente los pobladores del sector son los principales afectados.

De acuerdo con Méndez Veladia (2013), el principal agente de contaminación visual son los "carteles publicitarios" en todas sus presentaciones. La cantidad inmensurable de anuncios, todos de diferentes formas, tamaños y colores afecta a la salud de los seres humanos vulnerables a esto. Esta será la que se desarrolle en la presente investigación por ser parte del tema central de la misma.

La publicidad exterior afecta el entorno, es el agente más notorio por su efecto inmediato en la población, creando una sobre-estimulación en el ser humano mediante la información indiscriminada, y los múltiples mensajes que invaden la mirada. Esta situación no sólo atenta contra la belleza del espacio público, sino también sobre la lectura poco clara que tienen los individuos del mismo, dificultando la identificación del habitante con su paisaje El espacio público se encuentra desvirtuado e invadido por postes, carteles, y la lamentablemente la vegetación puede verse destruida. Este panorama es terriblemente agresivo para el hombre común, por lo que podemos imaginar cuánto lo es para un discapacitado, niño o anciano, ya que este grupo de individuos se podría considerar que pueden tener un mayor nivel de influencia por sus condiciones (Couto, 2007).

La contaminación visual publicitaria se agrava en tiempos de crisis económica, donde el achicamiento del mercado y la pelea por ganar espacios publicitarios conlleva la proliferación de anuncios ilegales y el abuso de la normativa vigente. La publicidad exterior trata de ser cada vez más atractiva y se apela a efectos tales como diseño, color, luz, movimiento, tamaño, siendo así un factor de distracción.

El tema de contaminación visual generada por el uso excesivo de publicidad es mundial, como se puede ver en varios países, que cada vez presta mayor atención a este problema. En varios países del mundo ya existen leyes o proyectos de ley sobre el uso de la publicidad en espacios públicos; en Argentina, en la ciudad de Rosario; en Costa Rica, existen leyes bastante estrictas que tienen en cuenta este tipo de contaminación.

La problemática de la contaminación visual provocada por la publicidad exterior fija podría sistematizarse en cuatro aspectos: cantidad (existen demasiados), tamaño (prácticamente no 
tiene límites), ubicación (se encuentran en cualquier lugar que uno mira), mensaje (muchas veces es de dudoso buen gusto o inapropiado para el medio en que se encuentra) (Couto, 2007).

En el Ecuador, no existe una legislación específica de contaminación visual, pero existen ciertos instrumentos legales que son aplicables en este caso; en la Constitución Ecuatoriana (2008), en el artículo 66 dice. "Se reconoce y garantizará a las personas: El derecho a vivir en un ambiente sano, ecológicamente equilibrado, libre de contaminación y en armonía con la naturaleza." Lo cual determina que las autoridades competentes deben velar por que la población disfrute de este derecho.

En la Ley Orgánica de Transporte Terrestre, Tránsito y Seguridad Vial (2008) en el Capítulo IV Del Ambiente, en la Sección 2: De la contaminación visual, dice: "Se prohíbe la instalación en carreteras de vallas, carteles, letreros luminosos, paneles publicitarios $u$ otros similares que distraigan a los conductores y peatones, afecten la seguridad vial, persuadan o inciten a prácticas de conducción peligrosa, antirreglamentaria o riesgosa." Este tipo de planteamientos permite regular el uso de publicidad en distintos ámbitos de la ciudad, por lo cual es importante tomarla en cuenta en lugares donde existe circulación vehicular constante.

En el Reglamento de la Ley de Transporte Terrestre, Tránsito y Seguridad Vial (2012) en el Capítulo III De la contaminación visual, dice: "Se prohíbe la instalación de rótulos tanto internos como externos que afecte la visibilidad del conductor y de los usuarios, salvo los que sean parte de la señalética de información e identificación autorizadas por la Agencia Nacional de Tránsito o por los GADs. Los agentes de tránsito estarán autorizados a retirar la rotulación no autorizada". Aunque la competencia de este control está establecida en este reglamento, la misma forma parte de las atribuciones que tiene el Municipio del Distrito Metropolitano de Quito.

Con los artículos anteriores se complementan con los artículos de las siguientes ordenanzas a ser explicadas.

En la Ordenanza 0330 del Distrito Metropolitano de Quito del año 2010, señala que es indispensable regular la utilización o el aprovechamiento del espacio público a través de la colocación de publicidad exterior en el Distrito Metropolitano de Quito, con el fin primordial de compatibilizar esta actuación con la protección de la seguridad ciudadana, la prevención de la contaminación ambiental, la protección, el mantenimiento y la mejora de los valores del paisaje urbano, y el buen uso del espacio público. En la misma Ordenanza, estipula: "El presente título tiene por objeto regular las condiciones a las que se sujetarán las instalaciones y el ejercicio de la actividad publicitaria exterior, cualquiera que sea el medio o sistema utilizado para la transmisión del mensaje, con el fin primordial de compatibilizar la colocación de la publicidad exterior con la seguridad de las personas, los bienes o el ambiente, y mantenimiento y mejora del ornato y paisaje del Distrito Metropolitano de Quito." Esta ordenanza es la única que regula el uso de 
publicidad en Quito, por lo cual será utilizada para la construcción de los indicadores de contaminación visual.

La problemática actual se centra en que no existe un medio adecuado de medición de contaminación visual, es un fenómeno de polución de características singulares, debido a que no se transmite a través de un vector sobre el que se pueda actuar, ya que este tipo de contaminación es subjetiva y depende, en su mayoría, de la percepción de las personas afectadas. Se observa que la publicidad exterior ha venido acrecentándose de manera paulatina, lo que significa un efecto negativo para el ambiente, aunque no se pude negar que la publicidad exterior es un medio generador de riqueza. Se manifiesta que los principales problemas que puede padecer una persona sujeta a contaminación visual son: stress, dolor de cabeza, mareos, ansiedad. También se puede indicar otros daños, como: distracciones peligrosas, especialmente al volante, problemas de atención, disminución de la eficiencia laboral, mal humor, trastornos de agresividad (Jerez Paredes, 2007).

Varios autores contribuyen a toda esta lista de efectos de la contaminación visual en la población, por ejemplo algunas otras incidencias de este tipo de contaminación son: alteraciones del sistema nervioso, impedimentos de transito libre y facilidad del mismo, la estética se ve afectada, desmejoramiento panorámico, problemas ecológicos (se rompe el equilibrio ecológico cuando algunas especies se alejan) y disminución de la eficiencia laboral, mal humor, trastornos de agresividad, cefaleas, etc. (Gallardo Pacheco, 2011).

Como se puede ver en la Figura 1, se detalla el trayecto donde se realizó el estudio, se puede observar la puntualización del "Inicio" y "Fin" de la distancia investigada. El área de estudio tiene una longitud de aproximadamente $800 \mathrm{mts}$.

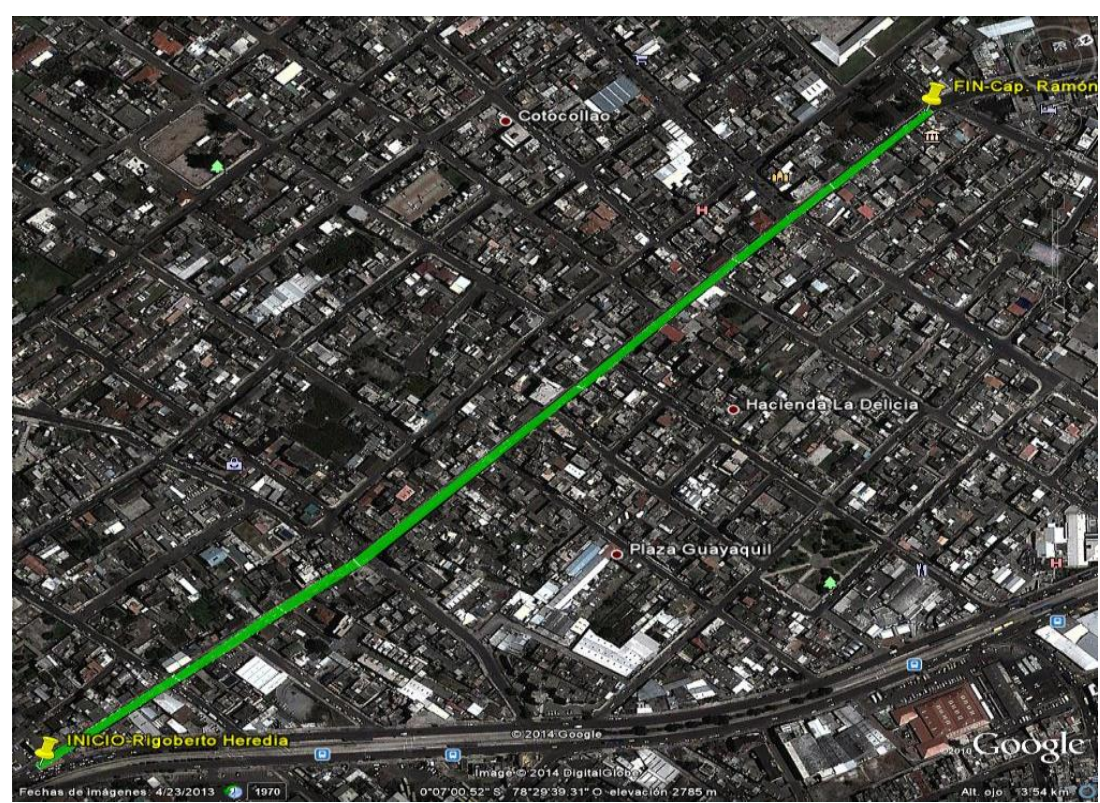

Figura 1. Tramo de la Investigación

(Google Earth, 2014) 


\section{Metodología}

La metodología que se aplicó en esta investigación englobó una relación entre el ambiente y la población, ya que al ser un sistema, depende de estas interacciones. La metodología que se usó debe comprender tanto aspectos cualitativos como cuantitativos para conocer la presencia de contaminación visual y los efectos que tiene en sus habitantes.

La investigación a realizar es de tipo no experimental descriptivo, ya que se realizó una investigación sin manipular deliberadamente las variables y se observaron los fenómenos tal y como se dan en su contexto natural para su posterior análisis; el uso de este tipo de investigación describió las relaciones existentes entre dos o más variables en un momento dado midiendo así dicha relación. El alcance de la presente investigación es realizar una estimación de los niveles de contaminación visual presente en el área de estudio así como los efectos que esta tiene sobre la población del sitio.

La presente investigación se realizó en el Barrio Cotocollao ubicado en la Parroquia Cotocollao que se encuentra en el Sector Zonal "La Delicia", específicamente desde la esquina de la calle Rigoberto Heredia hasta la calle Capitán Ramón Chiriboga, por las características que este trayecto posee.

Los cambios en la fisonomía y las actividades del sector, así como su ubicación, son algunas de las razones para la selección de este barrio como objeto para la investigación de la presencia de contaminación visual.

En el análisis de la contaminación visual y sus efectos en la población fue necesario utilizar varios tipos de investigación para conocer la situación actual con respecto al tema y a su vez ir profundizando en la misma. Por lo que se utilizó el método Deductivo Simple y el Combinado ya que en los mismos se unen tanto la investigación documental como la de campo. Se utilizó el tipo de Investigación documental para conocer el estado actual en el que se encuentra el análisis de la contaminación visual tanto a nivel internacional como nacional y así ir tomando guías para la realización de esta investigación. Posteriormente, se aplicó la investigación de campo para realizar una observación directa a los grupos y fenómenos, enfatizando en las variables a ser utilizadas en la investigación, es decir, analizar el área donde se manifiesta la contaminación visual y su relación con la población. Se realizó este tipo de investigación para que los datos requeridos sean recogidos de manera directa y así efectuar un análisis causa-efecto.

El análisis cuantitativo se centró en el estudio de las relaciones entre variables cuantificables (aplicación de indicadores de contaminación visual), mientras que el análisis cualitativo lo hace en contextos estructurales y situacionales (aplicación de encuestas y categorización de los efectos de este tipo de contaminación) lo que permitió analizar la perspectiva de la población frente a la problemática así como los efectos que la contaminación visual tiene el los habitantes. Con estos 
análisis se determinaron los indicadores de la contaminación visual y los niveles de la misma por la presencia de la publicidad exterior fija (rótulos publicitarios), así como los efectos que esta tiene sobre la población.

Para la aplicación de las encuestas se definió una muestra aleatoria de la población en el sector a analizar, partiendo del total de población en todo el barrio Cotocollao y no un especificado de la población total del sector a realizar la investigación, como se ve en la Ecuación 1:

$$
\begin{gathered}
n=(\text { total de casa*aproximado de habitantes por casa })^{*} 10 \% \\
n=\left(180^{*} 4\right)^{*} 10 \% \\
n=(720)^{*} 10 \% \\
n=72
\end{gathered}
$$

En la longitud del estudio existe un total de 180 casas (locales comerciales) aproximadamente, y se realizó una indagación de un aproximado de habitantes por vivienda, teniendo como resultado 4 personas aproximadamente. Se calculó el aproximado de $10 \%$ del total de la población para encontrar la muestra idónea. Por lo tanto, para que la muestra sea representativa, adecuada y válida, no se debe utilizar un número menor de 72 individuos.

Para la observación directa de los hechos, se llevó a cabo un registro fotográfico de los niveles de contaminación visual con base en la presencia del contaminante a ser analizado: Publicidad Exterior Fija (Rótulos y Vallas). Para el levantamiento de esta información se usó como instrumento la Ficha de Observación. Este instrumento consta de varias variables que serán de utilidad para el posterior análisis y triangulación con los resultados de los otros instrumentos utilizados, así como también fueron útiles como fuente primordial para la fabricación de los indicadores de contaminación visual. Como primera instancia, se dividió el tramo a ser analizado en partes iguales, como se presenta en la Figura 2, para que en cada una de estas sea aplicada una ficha de observación y así ir completando las variables a ser analizados (8 tramos de 100m cada uno).

La información en las fichas de observación requería realizar mediciones de la Publicidad Exterior Fija (vallas y rótulos) presente en cada tramo así como el número presente en cada vivienda, por lo tanto para completar las fichas de observación se realizó dos actividades: Medición del área de rótulo usando como equipo el distanciómetro, y el conteo directo de la cantidad de rotulación presente.

Las encuestas se las realizaron mediante un cuestionario, el mismo que proporciona un sistema de análisis de los efectos visuales útil y fácil de documentar. La encuesta estuvo compuesta de 6 partes: objetivo, conocimientos generales de la población, conocimiento de publicidad exterior fija, conocimiento de contaminación visual, efectos de este tipo de contaminación. Para la determinación de existencia de contaminación visual así como los niveles de la misma, se establecieron indicadores que sean de utilidad para la generación de información y de esta 
manera contrastar los resultados para su posterior análisis y finalmente dar conclusiones al respecto.

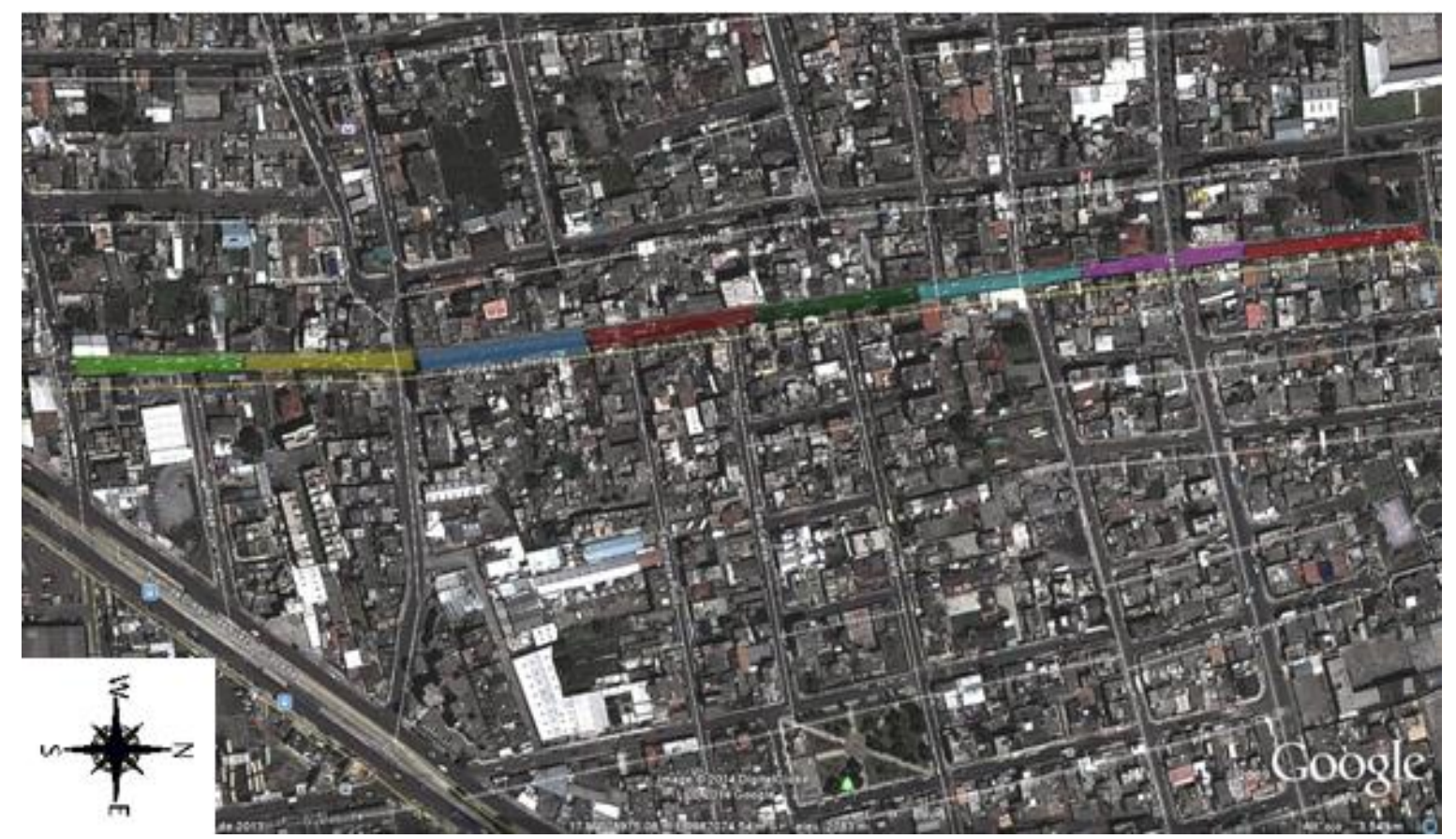

Figura 2. División de los tramos para aplicar Fichas de Observación

(Google Earth, 2014)

Para formular los indicadores, se estudiaron las reglamentaciones de varios países, y se analizó la información generada por los mismos. Esta información fue analizada y comparada, la misma ordenándose según sus coincidencias para el análisis de los indicadores de contaminación visual en otros países, tomando en cuenta la forma en cómo estos son aplicados: en general se definen por el número de vallas, ubicación, iluminación y cumplimiento de la normativa, entre otros. Tanto la información de la reglamentación como la existencia de indicadores de contaminación visual en otros países fueron realizadas con la investigación documental tanto física como digital.

Una vez realizada esta investigación se procedió a seleccionar la información y adaptación a nivel nacional teniendo en cuenta las características con que debe contar la misma como practicidad, factibilidad, comparabilidad y así proceder a la formulación de los indicadores para su aplicación local. Los indicadores diseñados dependerán de la cantidad de información disponible así como la profundidad que esta posea, para que de esta manera los resultados del estudio tengan mayor veracidad.

Se ha estructurado cinco indicadores básicos para el presente análisis de contaminación visual basándose principalmente en la Ordenanza Municipal 0330 "De la Licencia Metropolitana Urbanística de Publicidad Exterior - LMU (41)" del año 2010, donde existen reglas técnicas acerca de la publicidad exterior fija, los indicadores son: 
Indicador 1.- Porcentaje de rótulos por área.

$$
\text { Indicador Cont.Visual } 1=\frac{N^{\circ} \text { de rótulos }}{\text { Cada } 100 \mathrm{~m} \text { fachada }} \times 100 \%
$$

El Objetivo es contabilizar la cantidad de rotulación presente en el sector, estableciendo así tramos donde la magnitud de colocación de publicidad exterior fija sea excesiva e ir categorizando de lugares donde la rotulación sea mayor que otras.

Indicador 2.- Porcentaje de rótulos cuya área no cumple la norma.

$$
\text { Indicador Cont.Visual } 2=\frac{N^{\circ} \text { de rótulos con el área fuera de la normativa }}{N^{\circ} \text { de rótulos totales cada } 100 \mathrm{~m} .} \times 100 \%
$$

En la Ordenanza anteriormente descrita indica que la Publicidad Exterior Fija tiene un área determinada $\left(3 \mathrm{~m}^{2}\right)$.ya que si un rótulo tiene mayor área que la permitida, el efecto que tiene sobre la población será mayor. El objetivo de este indicador es analizar la cantidad de rotulación que tiene mayor área en comparación con el área permitida por la ley sin tener en cuenta el número de publicidad legal o ilegal. Este indicador ayuda a visualizar la cantidad de publicidad exterior fija (rótulos) que tiene un área fuera de la ley, por lo tanto, el nivel de contaminación visual y los efectos en la población serán mayor si se encuentra fuera de la normativa.

\section{Indicador 3.- Porcentaje de rótulos cuya distancia con la que se encuentran montados sobre la fachada no cumple la norma.}

$$
\text { Indicador Cont.Visual } 3=\frac{N^{\circ} \text { de rótulos montados fuera de la normativa }}{N^{\circ} \text { de rótulos totales cada } 100 \mathrm{~m} .} \times 100 \%
$$

En la Ordenanza hacer referencia a la distancia con la que se encuentra montada la Publicidad Exterior Fija, ya que si tienen una distancia mayor sobre la fachada $(20 \mathrm{~cm})$, el efecto que esto ocasiona en la población es mayor en comparación a que se encuentren montados lo más cerca posible sobre las viviendas. Al igual que el Indicador 2 "Porcentaje de rótulos que no cumplen la norma", la investigación de campo realizada es fundamental para este indicador.

Indicador 4.- Porcentaje de locales comerciales con más de un elemento publicitario.

Indicador Cont.Visual 4

$$
=\frac{N^{\circ} \text { de locales comerciales con más de un elemento publicitario }}{N^{\circ} \text { de locales totales comerciales }} \times 100 \%
$$

Según la Ordenanza 0330 que se describió con anterioridad cada sitio debe tener como máximo un elemento publicitario, ya que el exceso de publicidad exterior fija presente en un solo sitio comercial, tiene mayor efecto en la población en comparación a aquellos que tienen un solo elemento publicitario. Al igual que el anterior indicador, para la fabricación de este es necesario 
realizar un trabajo de campo previo, donde se realiza un conteo de la cantidad de publicidad exterior fija presente en cada local comercial del sector. El objetivo de este indicador es conocer el número de sitios comerciales que cumplen con la ordenanza 0330 de elementos publicitarios máximos permitidos por la ley y analizar los sectores del estudio de trabajo donde existe una proliferación de Publicidad Exterior Fija ocasionando mayor contaminación visual.

\section{Indicador 5.- Presencia de contaminación visual}

\section{Indicador Cont.Visual 5}

$$
=\frac{N^{\circ} \text { de personas que aceptan presencia de Contaminación Visual }}{N^{\circ} \text { total de personas encuestadas }} \times 100 \%
$$

La presencia de este tipo de contaminación es algo subjetivo, depende de la perspectiva de la población del lugar y del análisis de la presencia de contaminación visual presente producto de la Publicidad Exterior Fija del lugar (Rótulos). Para la fabricación de este indicador fue fundamental la realización de encuestas a la población.

El objetivo de este indicador es analizar la presencia de contaminación visual desde la perspectiva de la población del sitio a ser investigado. Una vez fabricados los indicadores se procedió a su aplicación para triangular la información y dar a conocer la presencia de contaminación visual y los niveles de la misma en el sector. Debido a la inexistencia de patrones cuantitativos para la evaluación de la gravedad de la contaminación visual y los efectos que esta tiene en la población, estas valoraciones necesitan la experiencia y juicio de profesionales cualificados para que puedan ser analizados.

\section{Resultados}

Los resultados de la aplicación del Indicador 1 "Porcentaje de rótulos por área" fue que en una longitud de $800 \mathrm{mts}$. Compuesta por las dos aceras que constituyen el área del estudio se observó que existen 269 rótulos, lo que refleja un uso del $34 \%$ de la totalidad del tramo del estudio (Tabla $1)$.

Los resultados de la aplicación del Indicador 2 "Porcentaje de rótulos cuya área no cumple la norma" fue que en la totalidad de la longitud de la investigación contabilizando la cantidad de rotulación cuya área no cumple la norma en las dos calles que lo conforman, de una totalidad de 269 rótulos fijos presentes, 154 rótulos fijos tienen el área fuera de la Ordenanza 0330; lo que significa que existe un total de 57\% de Publicidad Exterior Fija en el lugar con un área mayor a $3 \mathrm{mts}^{2}$ que es lo que establece la normativa lo que significa que más de la mitad de la rotulación presente tiene un área mayor por lo que la contaminación visual y los efectos que esta tienen son mayores en este tramo (Tabla 2). 
Tabla 1. Indicador 1 - Porcentaje de rótulos por área

\begin{tabular}{|l|c|c|}
\hline \multicolumn{2}{|c|}{ INDICADOR 1 - RESULTADOS TOTALES } \\
\hline $\begin{array}{c}\text { NÚMERO DE } \\
\text { TRAMO }\end{array}$ & $\begin{array}{c}\text { TOTAL DE } \\
\text { RÓTULOS }\end{array}$ & PORCENTAJE \\
\hline TRAMO 1 & 64 & $64 \%$ \\
\hline TRAMO 2 & 35 & $35 \%$ \\
\hline TRAMO 3 & 33 & $33 \%$ \\
\hline TRAMO 4 & 34 & $34 \%$ \\
\hline TRAMO 5 & 34 & $34 \%$ \\
\hline TRAMO 6 & 36 & $36 \%$ \\
\hline TRAMO 7 & 11 & $11 \%$ \\
\hline TRAMO 8 & 22 & $22 \%$ \\
\hline TOTAL & $\mathbf{2 6 9}$ & $\mathbf{3 4} \%$ \\
\hline
\end{tabular}

Tabla 2. Indicador 2 - Porcentaje de rótulos cuya área no cumple la norma

\begin{tabular}{|c|c|c|c|c|}
\hline \multicolumn{5}{|c|}{ INDICADOR 2 - RESULTADOS TOTALES } \\
\hline \multirow[t]{2}{*}{$\begin{array}{l}\text { NÚMERO DE } \\
\text { TRAMO }\end{array}$} & \multirow[t]{2}{*}{$\begin{array}{l}\text { TOTAL DE } \\
\text { RÓTULOS }\end{array}$} & \multicolumn{2}{|c|}{$\begin{array}{l}\text { ÁREA NORMATIVA } \\
\text { 3m2 } \\
\text { Ordenanza 0330; } \\
\text { Anexo Único 2.1.1 }\end{array}$} & \multirow[t]{2}{*}{$\begin{array}{c}\text { PORCENTAJE DE } \\
\text { NO } \\
\text { CUMPLIMIENTO }\end{array}$} \\
\hline & & $\mathrm{SI}$ & NO & \\
\hline TRAMO 1 & 64 & 27 & 37 & $58 \%$ \\
\hline TRAMO 2 & 35 & 20 & 15 & $43 \%$ \\
\hline TRAMO 3 & 33 & 15 & 18 & $55 \%$ \\
\hline TRAMO 4 & 34 & 14 & 20 & $59 \%$ \\
\hline TRAMO 5 & 34 & 16 & 18 & $53 \%$ \\
\hline TRAMO 6 & 36 & 17 & 19 & $53 \%$ \\
\hline TRAMO 7 & 11 & 0 & 11 & $100 \%$ \\
\hline TRAMO 8 & 22 & 6 & 16 & $73 \%$ \\
\hline TOTAL & 269 & 115 & 154 & $57 \%$ \\
\hline
\end{tabular}

Los resultados de la aplicación del Indicador 3 "Porcentaje de rótulos cuya distancia con la que se encuentran montados sobre la fachada no cumple la norma" fue que en la totalidad de la longitud de la investigación calculando la cantidad de rotulación cuya distancia con la que se encuentran montados sobre la fachada no cumple la norma en las dos calles que lo conforman, de una totalidad de 269 rótulos fijos presentes, 144 rótulos fijos se encuentran montados sobre la fachada a más de $20 \mathrm{~cm}$ siendo un $54 \%$ de la totalidad lo que significa que existe mayor nivel de contaminación visual y efectos en la población (Tabla 3).

Los resultados de la aplicación del Indicador 4 "Porcentaje de locales comerciales con más de un elemento publicitario" fue que en la totalidad de la longitud de la investigación contabilizando el número de locales comerciales en las dos calles que lo conforman que poseen más de un 
elemento publicitario por lo que no cumplen la norma, de una totalidad de 181 locales comerciales, 94 locales cuentan con más de una Publicidad Exterior Fija por lo que se encuentran fuera de la normativa; lo que significa un $52 \%$ de la totalidad de locales comerciales con más de un elemento publicitario lo que significa que existe mayor nivel de contaminación visual y efectos en la población al existir una proliferación de publicidad (Tabla 4).

Tabla 3. Indicador 3 - Porcentaje de rótulos cuya distancia con la que se encuentran montados sobre la fachada no cumple la norma

\begin{tabular}{|c|c|c|c|c|}
\hline \multicolumn{5}{|c|}{ INDICADOR 3 - RESULTADOS TOTALES } \\
\hline \multirow[t]{2}{*}{$\begin{array}{l}\text { NÚMERO } \\
\text { DE FICHA }\end{array}$} & \multirow[t]{2}{*}{$\begin{array}{l}\text { TOTAL DE } \\
\text { RÓTULOS }\end{array}$} & \multicolumn{2}{|c|}{$\begin{array}{c}\text { SOBRE FACHADA } \\
\text { NORMATIVA } \\
\text { max. } 20 \mathrm{~cm} \\
\text { Ordenanza 0330; } \\
\text { Anexo Único 2.1.1 }\end{array}$} & \multirow[t]{2}{*}{$\begin{array}{c}\text { PORCENTAJE } \\
\text { DE NO } \\
\text { CUMPLIMIENTO }\end{array}$} \\
\hline & & SI & NO & \\
\hline TRAMO 1 & 64 & 31 & 33 & $52 \%$ \\
\hline TRAMO 2 & 35 & 20 & 15 & $43 \%$ \\
\hline TRAMO 3 & 33 & 14 & 19 & $58 \%$ \\
\hline TRAMO 4 & 34 & 15 & 19 & $56 \%$ \\
\hline TRAMO 5 & 34 & 13 & 21 & $62 \%$ \\
\hline TRAMO 6 & 36 & 21 & 15 & $42 \%$ \\
\hline TRAMO 7 & 11 & 3 & 8 & $73 \%$ \\
\hline TRAMO 8 & 22 & 8 & 14 & $64 \%$ \\
\hline TOTAL & 269 & 125 & 144 & $54 \%$ \\
\hline
\end{tabular}

Tabla 4. Indicador 4 - Porcentaje de locales comerciales con más de un elemento publicitario

\begin{tabular}{|c|c|c|c|c|}
\hline \multicolumn{5}{|c|}{ INDICADOR 4 - RESULTADOS TOTALES } \\
\hline \multirow[t]{2}{*}{$\begin{array}{l}\text { NÚMERO } \\
\text { DE FICHA }\end{array}$} & \multirow[t]{2}{*}{$\begin{array}{l}\text { NÚMERO DE } \\
\text { LOCALES } \\
\text { COMERCIALES } \\
\text { TOTALES }\end{array}$} & \multicolumn{2}{|c|}{$\begin{array}{c}\text { NÚMERO DE } \\
\text { CASAS CON MÁS } \\
\text { DE UNA } \\
\text { PUBLICIDAD } \\
\text { Ordenanza 0330; } \\
\text { Anexo Único 2.1.1 }\end{array}$} & \multirow[t]{2}{*}{$\begin{array}{c}\text { PORCENTAJE DE } \\
\text { NO } \\
\text { CUMPLIMIENTO }\end{array}$} \\
\hline & & SI & NO & \\
\hline TRAMO 1 & 35 & 13 & 22 & $63 \%$ \\
\hline TRAMO 2 & 20 & 11 & 9 & $45 \%$ \\
\hline TRAMO 3 & 21 & 10 & 11 & $52 \%$ \\
\hline TRAMO 4 & 24 & 11 & 13 & $54 \%$ \\
\hline TRAMO 5 & 28 & 13 & 15 & $54 \%$ \\
\hline TRAMO 6 & 22 & 11 & 11 & $50 \%$ \\
\hline TRAMO 7 & 11 & 9 & 2 & $18 \%$ \\
\hline TRAMO 8 & 20 & 9 & 11 & $55 \%$ \\
\hline TOTAL & 181 & 87 & 94 & $52 \%$ \\
\hline
\end{tabular}

Los resultados de la aplicación del Indicador 5 "Presencia de contaminación visual" de la muestra total de la aplicación de las encuestas (72 encuestados), 60 personas encuestados dieron como respuesta que en su barrio existe contaminación visual como resultado de un exceso de 
Publicidad Exterior Fija, lo que representa que el $83 \%$ de ellos afirman la presencia de contaminación visual (Tabla 5).

Tabla 5. Indicador 5 - Presencia de contaminación visual

\begin{tabular}{|c|c|c|c|}
\hline \multicolumn{4}{|c|}{ INDICADOR 5 - RESULTADOS ENCUESTA } \\
\hline \multirow{2}{*}{$\begin{array}{c}\text { NÚMERO } \\
\text { TOTAL DE } \\
\text { PERSONAS } \\
\text { ENCUESTADAS }\end{array}$} & \multicolumn{2}{|c|}{$\begin{array}{c}\text { RESPUESTAS DE } \\
\text { PERSONAS } \\
\text { ENCUESTADAS }\end{array}$} & \multirow{2}{*}{$\begin{array}{l}\text { PORCENTAJE DE } \\
\text { PRESENCIA DE } \\
\text { cVISUAL }\end{array}$} \\
\hline & SI & NO & \\
\hline 72 & 60 & 12 & $83 \%$ \\
\hline
\end{tabular}

Otro de los resultados fue analizar los puntos críticos con respecto a contaminación visual en el sitio de la investigación; para este análisis se tomaron en consideración los resultados de los Indicadores 2, 3 y 4 ya que el objetivo fue analizar y categorizar los tramos (8 tramos) del sitio según el nivel de contaminación visual existente.

Una vez analizados los tramos con los tres indicadores, es necesario realizar un cálculo para poder categorizar dependiendo del nivel de contaminación visual existente, siendo el cálculo el siguiente:

Cálculo del Nivel de Contaminación Visual del Tramo $x$

$$
=\frac{(\% \text { Indicador } 2+\% \text { Indicador } 3+\% \text { Indicador } 4)}{3}
$$

Teniendo así en el caso del Tramo 1 y observando la Tabla 1:

$$
\text { Cálculo del Nivel de Contaminación Visual del Tramo } 1=\frac{(58 \%+52 \%+63 \%)}{3}
$$

Una vez aplicada el anterior cálculo, se categorizó de acuerdo a los porcentajes de contaminación visual (descendente) de acuerdo a la Tabla 6.

Tabla 6. Categorización del porcentaje de contaminación visual por Tramos

\begin{tabular}{|r|c|c|}
\cline { 2 - 3 } \multicolumn{1}{c|}{} & TRAMO & $\%$ \\
\hline 1 & TRAMO 7 & 64 \\
\hline 2 & TRAMO 8 & 64 \\
\hline 3 & TRAMO 1 & 58 \\
\hline 4 & TRAMO 4 & 56 \\
\hline 5 & TRAMO 5 & 56 \\
\hline 6 & TRAMO 3 & 55 \\
\hline 7 & TRAMO 6 & 48 \\
\hline 8 & TRAMO 2 & 44 \\
\hline
\end{tabular}


Para la categorización de los tramos cuyo resultado tuvo los mismos valores, se analizó a profundidad el Indicador 2: Área total de rotulación, ya que si los rótulos tienen mayor área el impacto será mayor.

Por lo tanto, los puntos críticos serán principalmente el tramo séptimo y el octavo al tener $64 \%$ de contaminación visual, seguidos por los tramos cuarto, quinto y tercero, que tienen más del $50 \%$ de contaminación visual existente.

Solo los tramos sexto y segundo poseen un nivel de contaminación visual menor al 50\%, por lo que no fueron considerados puntos críticos en comparación a los otros tramos.

Para realizar una estimación de los niveles de contaminación visual en el sitio de investigación es necesario tener una escala. Esta escala se la realizó en base a la experiencia de esta investigación, así como de los datos emitidos una vez aplicados los indicadores.

Por lo tanto, la escala que se observa en la Tabla 7, para la categorización de los niveles de contaminación visual:

Tabla 7. Escala de los Niveles de Contaminación visual

\begin{tabular}{|l|l|}
\hline \multicolumn{2}{|c|}{$\begin{array}{c}\text { ESCALA DE LOS NIVELES DE } \\
\text { CONTAMINACIÓN VISUAL }\end{array}$} \\
\hline $100 \%-75 \%$ & Alto \\
\hline $75 \%-50 \%$ & Medio- Alto \\
\hline $50 \%-25 \%$ & Medio \\
\hline $25 \%-1 \%$ & Bajo \\
\hline
\end{tabular}

Una vez diseñada la escala de contaminación visual se prosigue con la estimación de los niveles de contaminación visual en cada uno de los tramos de acuerdo con los resultados de los Indicadores 2, 3 y 4, por consiguiente obteniéndose los valores de la Tabla 8.

Tabla 8. Estimación de niveles de contaminación visual en el sitio de estudio

\begin{tabular}{|c|c|c|c|}
\hline & TRAMO & $\%$ & NIVEL \\
\hline 1 & TRAMO 7 & 64 & \\
\hline 2 & TRAMO 8 & 64 & 은 \\
\hline 3 & TRAMO 1 & 58 & '́ \\
\hline 4 & TRAMO 4 & 56 & 무 \\
\hline 5 & TRAMO 5 & 56 & \\
\hline 6 & TRAMO 3 & 55 & \\
\hline 7 & TRAMO 6 & 48 & 음 \\
\hline 8 & TRAMO 2 & 44 & \\
\hline
\end{tabular}


Presencia de contaminación visual realizando una estimación de los niveles de este tipo de contaminación, se observa que todo el sector tiene nivel medio-alto de contaminación visual, inclusive los tramos sexto y segundo, que tiene un nivel de contaminación medio (Figura 3). Los efectos que tiene la contaminación visual en la población dependen de la perspectiva de ellos ya que se encuentran expuestos a este tipo de contaminación, por lo tanto, los resultados del cuestionario fueron claves.

\section{ESTIMACIÓN DE LOS NIVELES DE CONTAMINACIÓN VISUAL}

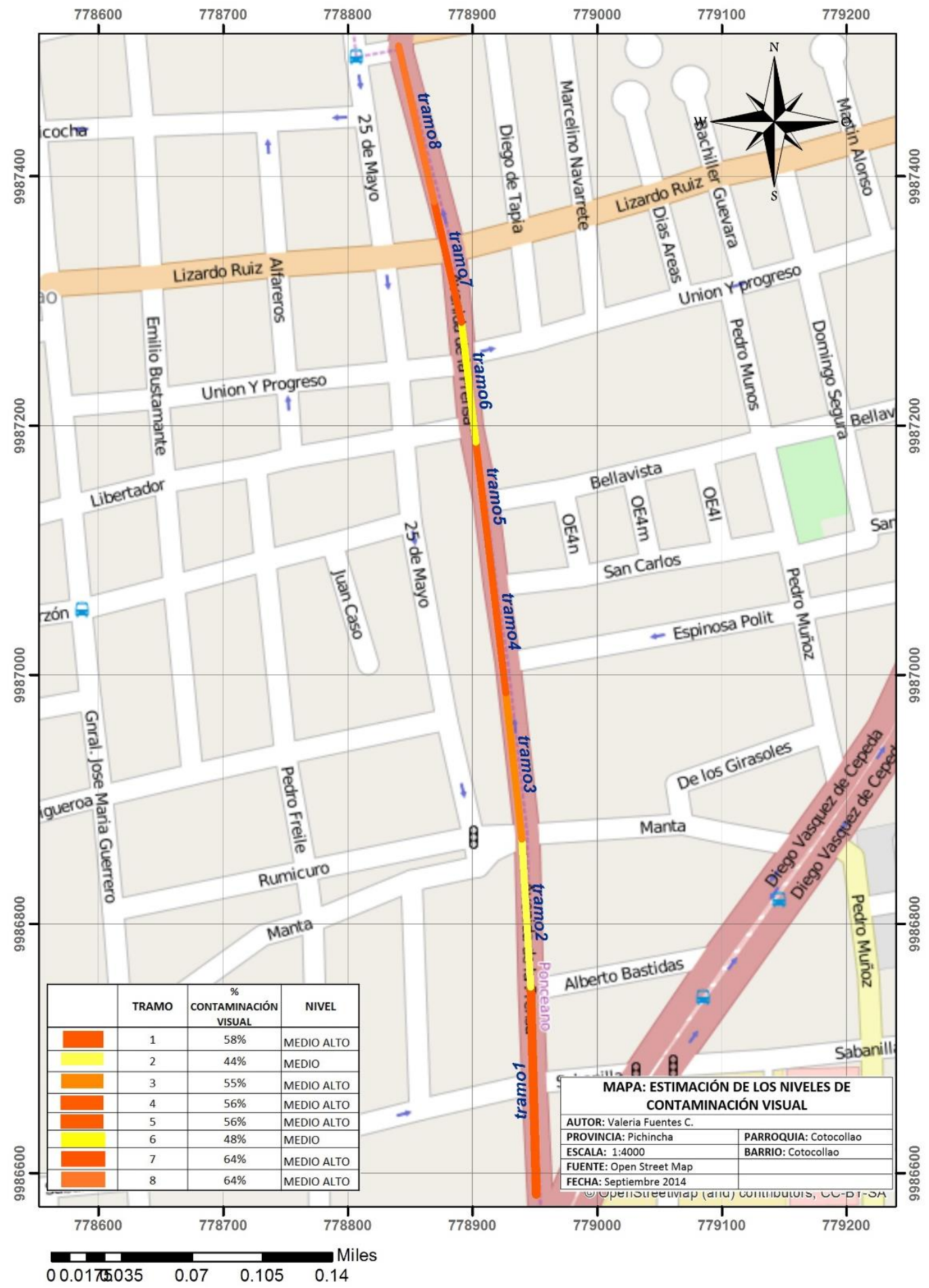

Figura 3. División de los tramos para aplicar Fichas de Observación (Google Earth, 2014) 
La opinión de las personas acerca de la contaminación visual como producto de la publicidad, se empezó a analizar los efectos que a su opinión generan este tipo de contaminación, teniendo así los resultados de la Tabla 9.

Tabla 9. Efectos de la contaminación visual

\begin{tabular}{|c|c|c|c|}
\hline \multicolumn{4}{|c|}{ 9. EFECTOS DE CONTAMINACIÓN VISUAL } \\
\hline & & NÚMERO & PORCENTAJE \\
\hline \multirow{9}{*}{ 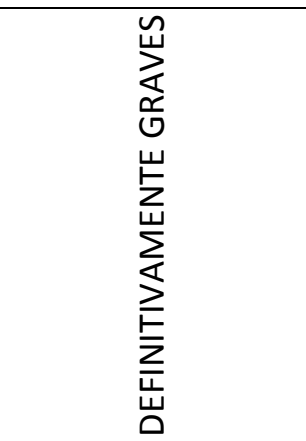 } & ESTRÉS & 67 & $93 \%$ \\
\hline & DOLOR DE CABEZA & 68 & $94 \%$ \\
\hline & CANSANCIO VISUAL & 70 & $97 \%$ \\
\hline & AMENAZA & 21 & $29 \%$ \\
\hline & PERTURBACIÓN & 30 & $42 \%$ \\
\hline & OFENSA & 24 & $33 \%$ \\
\hline & INCOMODIDAD & 59 & $82 \%$ \\
\hline & AGRESIÓN & 35 & $49 \%$ \\
\hline & DISTRACCIONES PELIGROSAS & 64 & $89 \%$ \\
\hline \multirow{2}{*}{$\begin{array}{c}\text { POTENCIALMENTE } \\
\text { GRAVES }\end{array}$} & PÉRDIDA EN LOS VALORES ESCÉNICOS & 58 & $81 \%$ \\
\hline & $\begin{array}{llll}\begin{array}{l}\text { AUMENTO } \\
\text { COMERCIAL }\end{array} & \text { EN } & \text { LA } & \text { ACTIVIDAD } \\
\end{array}$ & 60 & $83 \%$ \\
\hline \multirow{2}{*}{ 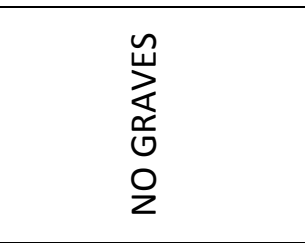 } & \begin{tabular}{llll|} 
OBSTRUCCIÓN & VISUAL & (FACHADAS, \\
ESPACIOS PÚBLICOS, & VÍAS DE \\
CIRCULACIÓN PEATONAL) & & \\
\end{tabular} & 68 & $94 \%$ \\
\hline & $\begin{array}{l}\text { BLOQUEO O INTERRUPCIÓN VISUAL } \\
\text { DEL PAISAJE NATURAL }\end{array}$ & 59 & $82 \%$ \\
\hline \multicolumn{2}{|r|}{ OTROS } & 0 & $0 \%$ \\
\hline \multicolumn{2}{|c|}{ NINGUNO DE LOS ANTERIORES } & 0 & $0 \%$ \\
\hline \multicolumn{3}{|c|}{ TOTAL ENCUESTADOS } & 72 \\
\hline
\end{tabular}

Los efectos que sufre la población, son tanto definitivamente graves, potencialmente graves y no graves, siendo el efecto que menor porcentaje tuvo "amenaza" con un $29 \%$ del total de los encuestados, y los efectos que tuvieron mayor incidencia fueron: Cansancio visual $97 \%$, dolor de cabeza 94\%, obstrucción visual 94\%, estrés 93\%, distracciones peligrosas $89 \%$, incomodidad $82 \%$, bloqueo o interrupción del paisaje natural $82 \%$ y pérdida en los valores escénicos $81 \%$.

\section{Discusión}

De acuerdo Méndez Velandia (2013), en el análisis de contaminación visual en Venezuela, se coincide con la existencia de esta problemática, especialmente por la presencia de publicidad tanto rótulos como vallas, denotando los efectos en la población. Aunque la contaminación visual no es un tipo de contaminación convencional, tal como la contaminación al agua o al suelo, la construcción de indicadores que permitan medir el cumplimiento de las normas (que son creadas para el bienestar de los ciudadanos, y evitar efectos tanto en el ambiente como en los seres humanos), son de gran importancia para el análisis de sus efectos en la población y su derecho a vivir en un ambiente sano. 
Al ser una fuente de contaminación visual el mal uso de publicidad exterior fija, el control por parte de las autoridades, en los sectores cuyos niveles estimados de este tipo de contaminación son considerados como críticos, debe tener un monitoreo constante, para de esta manera reducir los sus efectos mediante el cumplimiento de la normativa; así como también para una reducción de contaminación visual, especialmente en sectores donde ha aumentado la actividad comercial.

\section{Conclusiones}

Se concluyó que la población tiene varias nociones sobre la definición de la misma que son tanto afectaciones a la salud como a su entorno, de igual manera un exceso de elementos ajenos al ambiente, lo cual se constata que se relaciones de gran manera con las definiciones expuestas por varios autores.

Como aporte de esta investigación se propone un concepto que englobe todas las definiciones anteriormente descritas de contaminación visual, siendo el siguiente:

contaminación visual aquel tipo de contaminación que es de carácter urbano que es ocasionada por el uso excesivo de varios elementos ajenos al ambiente que alteran la estética y la imagen del paisaje ya sea natural o artificial, que afecta tanto a las condiciones, calidad de vida y las funciones vitales de los seres vivos.

Posterior a tener un concepto claro de contaminación visual, y a las investigaciones realizadas alrededor de la misma, se analizó la forma en que la contaminación visual puede ser medida, por lo que se creó cinco indicadores y los mismos puedan arrojar información cuantitativa de esta contaminación. Estos indicadores fueron aplicados en las dos aceras en cada tramo del área del estudio y de igual manera en todo el sector (Esquina de la Calle Rigoberto Heredia hasta la Calle Capitán Ramón Chiriboga), para tener referencias globales del nivel de contaminación existente.

Se concluye de los resultados de la aplicación de los indicadores y de realizar una estimación de los niveles de contaminación visual presente y conocer los puntos críticos existentes en el área de estudio se prosiguió a realizar estimaciones de los niveles de contaminación visual existente, donde se basó en una escala realizada a base de la experiencia de esta investigación, siendo los niveles del sector "Medio-Alto" seis de los tramos totales (el $75 \%$ del sector total) y dos como "Medios" (el 25\% del sector total).

Para finalizar, se analizó los efectos que tiene la contaminación visual en la población los mismos que se basaron en la percepción de la población frente a esta problemática. Los efectos que mayor incidencia que los pobladores opinaron que sufren a causa de este tipo de contaminación fueron: cansancio visual, dolor de cabeza, obstrucción visual, estrés, distracciones peligrosas, incomodidad, bloqueo del paisaje natural y pérdida de los valores escénicos. 


\section{Bibliografía}

Constitución de la República (2008)

Couto, M. M. (2007). Contaminación visual del paisaje, cartelería publicitaria en rutas. Metodología de la Investigación. Costa Rica: Maestría en Paisajismo-Instituto Universitario Bios.

Gallardo Pacheco, R. F. (2011). Propuesta de regulación del diseño publicitario de rotulación en el Centro Histórico de la ciudad de Latacunga para combatir la contaminación visual existente. Latacunga, Ecuador.

Jérez Paredes, M. T. (2007). Eficacia de las medidas legales existentes para evitar la contaminación visual en la ciudad de Guatemala. Guatemala: USAC.

Ley Orgánica de Transporte Terrestre, Tránsito y Seguridad Vial (2008)

Méndez Velandia, C. A. (2013). La contaminación visual de espacios públicos en Venezuela., El Caribe, España y Portugal: Red de Revistas Científicas de América Latina, pp. 45-60.

Municipio del Distrito Metropolitano de Quito (2010) Ordenanza Metropolitana 0330: De la Licencia Metropolitana Urbanística de Publicidad Exterior - LMU (41). Quito: MDMQ.

Municipio del Distrito Metropolitano de Quito (2012) Ordenanza Metropolitana 0310: "La Ordenanza Metropolitana Reformatoria de la Ordenanza Metropolitana No. 0330, sancionada el 23 de noviembre de 2010, que establece el régimen administrativo de la Licencia Metropolitana Urbanística de Publicidad Exterior LMU - (41). Quito: MDMQ.

Municipio del Distrito Metropolitano de Quito (2003): Ordenanza Sustitutiva a la Ordenanza de Zonificación No. 008 que contiene el plan de uso y ocupación del suelo (PUOS), recuperado en agosto del 2014 de:

http://www7.quito.gob.ec/mdmq ordenanzas/Ordenanzas/ORDENANZAS\%20A\%C3\%910S \%20ANTERIORES/ORDZ-011\%20-\%20PUOS\%20-\%20SUSTITUYE\%20ORMZ-008.pdf

Reglamento de la Ley de Transporte, Tránsito y Seguridad Vial (2012). 